\title{
SMOOTHING LOCALLY FLAT IMBEDDINGS ${ }^{1}$
}

BY R. C. KIRBY

Communicated by E. Dyer, August 23, 1965

The fundamental imbedding problem for manifolds is to classify the imbeddings of an $n$-manifold into a $q$-manifold under ambient isotopy. We announce here that the differentiable and topological cases of this problem for differentiable manifolds are the same if $2 q>3(n+1)$ and $q \geqq 8$.

This follows from Theorem 2 below which states that a locally flat imbedding of a compact differentiable manifold $M^{n}$ into a differentiable manifold $Q^{a}$ is ambient isotopic to a differentiable imbedding if $2 q>3(n+1)$ and $q \geqq 8$. Since this ambient isotopy may be chosen arbitrarily close to the identity map, the set of differentiable imbeddings is dense in the set of locally flat imbeddings of $M^{n}$ in $Q^{a}$.

It will then follow that two locally flat imbeddings of $M^{n}$ into $Q^{q}$ are ambient isotopic if they are homotopic; hence the classification problem reduces to a problem in homotopy theory.

Theorem 1. Let $f: B^{n} \rightarrow \operatorname{int} Q^{a}$ be a locally flat imbedding of the unit $n$-ball into $Q^{q}$. Such an $f$ always extends to $f: R^{q} \rightarrow$ int $Q^{q}$. Let $C^{n-1}$ be a compact differentiable submanifold of $\partial B^{n}=S^{n-1}$, and suppose that $f$ is differentiable on a neighborhood of $C^{n-1}$ in $B^{n}$. Let $q \geqq 7,2 q>3(n+1)$ and $\epsilon>0$. Then there exists an ambient $\epsilon$-isotopy $F_{t}: Q^{a} \rightarrow Q^{a}, t \in[0,1]$, satisfying

(1) $F_{0}=$ identity,

(2) $F_{1} f$ is differentiable on int $B^{n}$ and on a neighborhood of $C^{n-1}$ in $B^{n}$,

(3) $F_{t}=$ identity on $Q-N_{\epsilon}\left(f\left(B^{n}\right)\right)$ and on $f\left(R^{n}-\operatorname{int} B^{n}\right)$ for all $t \in[0,1]$,

(4) $\left|F_{t}(x)-x\right|<\epsilon$ for all $x \in Q^{a}$ and $t \in[0,1] .\left(N_{\epsilon}(X)\right)$ is the set of points within $\epsilon$ of $X$.)

THEOREM 2. Let $f: M^{n} \rightarrow Q^{a}$ be a locally flat imbedding such that either $f\left(M^{n}\right) \subset$ int $Q^{a}$ and $q \geqq 7$ or $f^{-1}\left(\partial Q^{a}\right)=\partial M^{n}$ and $q \geqq 8$. Let $2 q>3(n+1)$ and $\epsilon>0$. Then there exists an ambient $\epsilon-i$ sotopy $F_{t}: Q^{q} \rightarrow Q^{q}, t \in[0,1]$, satisfying

(1) $F_{0}=$ identity,

(2) $F_{1} f$ is a differentiable imbedding,

1 This is an announcement of a portion of the author's dissertation at the University of Chicago written under Professor Eldon Dyer. 
(3) $F_{t}=$ identity on $Q-N_{\epsilon}\left(f\left(M^{n}\right)\right)$ for all $t \in[0,1]$,

(4) $\left|F_{t}(x)-x\right|<\epsilon$ for all $x \in Q^{q}$ and $t \in[0,1]$.

The proof follows from Theorem 1 by considering the handlebody decomposition of $M^{n}$, and smoothing the imbedding of one handle at a time.

Only imbeddings of $M^{n}$ into $Q^{q}$ satisfying $f\left(M^{n}\right) \subset$ int $Q^{q}$ or $f^{-1}\left(\partial Q^{a}\right)=\partial M^{n}$ will be considered. Let $T$ be the set of equivalence classes of locally flat imbeddings of $M^{n}$ into $Q^{q}$ under equivalence by ambient isotopy. Similarly, let $D(C)$ be the set of equivalence classes of differentiable (combinatorial) imbeddings of $M^{n}$ into $Q^{q}$ under equivalence by ambient diffeotopy (ambient combinatorial isotopy). Let $H$ be the homotopy classes of locally flat imbeddings of $M^{n}$ into $Q^{q} . H$ is a subset of $\left[M^{n}, Q^{q}\right]$, the homotopy classes of maps of $M^{n}$ into $Q^{q}$. Then we have the following commutative diagram where the maps are the natural projections.

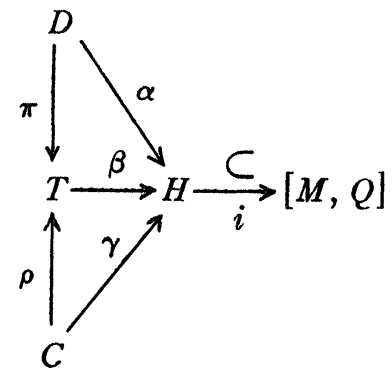

$\beta$ is clearly onto for all $n$ and $q$. Gluck has shown [1] that $\rho$ and $\gamma$, and hence $\beta$ and $\beta i$ are isomorphisms for $q \geqq 2 n+2$. Haefliger has shown [2] that $\pi$ is a monomorphism and that $\alpha$ is an isomorphism if $2 q>3(n+1)$.

It follows from Theorem 2 that $\pi$ is also epimorphic if $2 q>3(n+1)$ and either $q \geqq 7$ when $f\left(M^{n}\right)$ Cint $Q^{a}$ or $q \geqq 8$ when $f^{-1}\left(\partial Q^{q}\right)=\partial M^{n}$. Then $\pi$ and $\beta$ are isomorphisms in this range of dimensions.

\section{REFERENCES}

1. H. Gluck, Embeddings in the trivial range, Ann. of Math. 81 (1965), 195-210.

2. A. Haefliger, Plongements differentiables de variêtés dans varietés, Comment. Math. Helv. 36 (1961), 47-82.

University of California, Los Anget.es 